\title{
Queue and Power Control for Rechargeable Sensor Networks under SINR Interference Model
}

\author{
Zhoujia Mao, Can Emre Koksal, Ness B. Shroff \\ E-mail:maoz@ece.osu.edu,koksal@ece.osu.edu, shroff@ece.osu.edu
}

\begin{abstract}
Renewable energy sources can be attached to sensor nodes to substantially improve the performance of sensor networks. In networks with renewable energy sources, conservative energy expenditure may lead to missed recharging opportunities due to the batteries being full, while aggressive usage of energy may cause the network to be intermittently disconnected and reduction in coverage. Thus, new techniques and algorithms must be developed for networks with replenishment to balance these seemingly contradictory goals. In this paper, we consider an uplink wireless senor network with renewable energy sources attached to sensor nodes under the SINR interference model, where the achievable transmission rate of each sensor node depends on its SINR. We develop a provably efficient solution to maximize the total sensing rate subject to quality of service constraints.
\end{abstract}

\section{INTRODUCTION}

Wireless sensor networks have many applications in monitoring, maintenance, and environment sensing [1], [2], [3]. New developments in the area of renewable energy [4], [5] will help facilitate earlier and more wide-spread deployment of these networks by increasing their operational capacity and lifetime. These renewable sources of energy could be attached to the nodes, and would typically replenish the batteries at a slow rate (e.g., compared to the rate at which energy is consumed by a continuous stream of packet transmissions) that could be variable and dependent on the surroundings.

Unlike traditional battery-limited sensor networks, in networks with replenishment, the network lifetime can be infinity, and a node with a full battery may not be necessarily desirable. This is because a node with a full battery implies missed recharging opportunities, which in turn implies a reduced feasible sensing and transmission rates. On the other hand, an over aggressive use of energy may lead to lack of coverage or connectivity for certain time periods, or result in the network being incapable of transferring time-sensitive data to the sink because certain nodes have depleted their batteries. Thus, new techniques and algorithms must be developed for networks with replenishment to balance these seemingly contradictory goals. In this paper, we develop a joint rate and power control algorithm, which is provably efficient for rechargeable networks. We use the so-called "physical interference model," for which the transmission rate is a function of the signal to interference and noise ratio (SINR) and influenced by the power vector over the entire network.

While the problem of energy management in wireless networks has seen considerable attention, there have been few works [6], [7], [8], [9], [10], [11] that also include energy replenishment. In [6], [7], [8], [9] the authors study the problem of dynamic node activation, energy harvesting, sleepwake scheduling, and duty cycle controlling respectively under rechargeable networks. In [10] and [11], motivated by [12], the authors consider jointly managing the data and battery buffers to maximize the throughput with renewable energy sources under protocol interference model. The coupling between the data and battery buffers is what makes the problem notoriously difficult to solve using standard optimization based approaches. For example, unlike [12], which utilizes the fact that a static allocation policy is optimal, it is not even clear whether an optimal policy would be static in a rechargeable network setting. Specifically, with a battery buffer, there is an additional energy constraint that the allocated energy should be within the battery state, and this constraint is even more difficult than the average power constraint. In [10], the authors consider infinite data buffer and finite battery buffer sizes. They assume that the replenishing process is i.i.d, and show that the probability of battery state being less than the peak power or close to the full battery state vanishes as the battery size grows with their algorithm.

All the prior work on networks with replenishing nodes rely on discrete interference models, in order to develop practical solutions to the optimal resource allocation problem. Despite their simplicity, discrete interference models could be poor approximations to the actual interference, observed in wireless networks. On the other hand, physical interference models such as the SINR model are known to more accurately capture the impact of the interference more closely. Prior work on resource allocation and utility optimization under the SINR model include [13], [14], [15], [16], [17], [18]. In [13], [14], [15], the authors formulate optimization problems with QoS constraints on SINR. In [16], [17], [18], the authors consider an optimal power allocation problem such that a utility function of SINR is maximized. None of these studies consider energy replenishment. In this paper, we consider the power allocation problem combined with rate control, to achieve the maximal sensing rate with energy replenishment using the SINR model.

The main contributions of the paper can be summarized as follows:

- We develop a mathematical framework to handle the coupling between the data and battery queues that exists in networks with replenishment. Our mathematical framework allows does not require future knowledge of the data and replenishment. 
- Unlike existing works on utility maximization and network optimization, in our framework, we allow for a general arrival and replenishment process that does not require stationarity or ergodicity. This is especially important in such networks, because the replenishment profiles are typically non-stationary.

- We develop a provable efficient algorithm that achieves the optimum solution within a small gap under the SINR interference model.

\section{System Model and Problem Formulation}

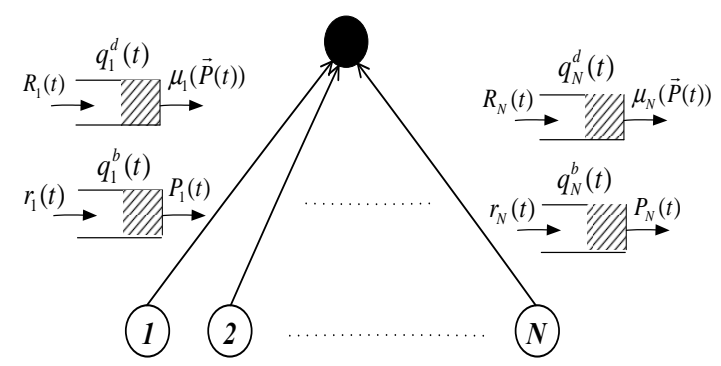

Fig. 1. Network Model

We consider a rechargeable sensor network with one base station and $N$ sensor nodes, as illustrated in Figure. 1. Sensor nodes $n \in \mathcal{N}=\{1,2, \ldots, N\}$ are attached to independent renewable power sources for replenishment. We assume a time slotted system. In time slot $t, A_{n}(t)$ and $R_{n}(t)$ denote the amount of available data for sensing and the actual amount of sensed data, at node $n$ that are destined to the base station. Hence, $R_{n}(t) \leq A_{n}(t), \forall t \geq 0, \forall n \in \mathcal{N}$. Let $r_{n}(t)$ denote the amount of replenishment at node $n$ in slot $t$. We assume that $0 \leq A_{n}(t) \leq A_{\max }<\infty$ and $0<r_{\min } \leq r_{n}(t) \leq r_{\max }<$ $\infty, \forall t \geq 0, \forall n \in \mathcal{N}$. We assume that each sensor node $n$ maintains an infinite size data buffer with state $q_{n}^{d}(t)$, and also maintains a finite battery buffer with size $B_{n}^{b}<\infty$ and state $q_{n}^{b}(t)$. Note that we focus on infinite data buffer size and finite battery buffer size for ease of illustration. Following our approach in [11], we can also allow for finite battery and data buffers. Vector $\vec{P}(t)=\left\{P_{1}(t), P_{2}(t), \ldots, P_{N}(t)\right\}$ denotes the power assignments over all the uplink communication pairs at time $t$. With this power allocation, the achieved transmission data rate for node $n$ is $\mu_{n}(\vec{P}(t))$. Let $\vec{P}$ be the sequence of power assignments $\{\vec{P}(0), \vec{P}(1), \ldots, \vec{P}(T-1), \ldots\}$ and $\vec{R}$ be the sequence of sensing rates $\{\vec{R}(0), \vec{R}(1), \ldots, \vec{R}(T-1), \ldots\}$. The power used by any user node in each slot is also upper bounded by the peak power $P_{\text {peak }} \ll \min _{n} B_{n}^{b}$. The rate-power function is assumed to have the following form under the SINR interference model:

$$
\mu_{n}(\vec{P}(t))=\log \left(1+\frac{g_{n} P_{n}(t)}{N_{n}+\sum_{i \in \mathcal{N}, i \neq n} g_{i} P_{i}(t)}\right)
$$

where $g_{n}$ is the path gain from the sensor node $n$ to the base station, and $N_{n}$ is the background noise at node $n$. Note that, even though $\log (\cdot)$ is a concave function, $\mu_{n}(\vec{P}(t))$ is not a concave function of $\vec{P}(t)$. We can see from this rate-power function that the transmission rate of node $n$ is influenced by the power assignment on all other nodes (interference) based on the SINR model.

Definition 1: $p_{n}^{o}$ is the long-term battery discharge ratio for node $n$ :

$$
p_{n}^{o}=\limsup _{T \rightarrow \infty} \frac{\sum_{t=0}^{T-1} 1_{n}^{o}(t)}{T},
$$

where

$$
\begin{aligned}
l_{n}^{o}(t)= & \text { indicator that battery is discharged } \\
& \text { in slot } t \text { for node } n \\
= & \begin{cases}0 & \text { if } q_{n}^{b}(t)-P_{n}(t)>0 \\
1 & \text { otherwise }\end{cases}
\end{aligned}
$$

Definition 1 is a suitable QoS metric for rechargeable sensor networks that represents how often the battery is discharged. Some applications may require the battery state to be always above certain positive level, then Definition 1 can be easily modified to represent how often the battery is below the desired level, and our solution structure works as well. Note that the ratio $p_{n}^{o}$ represents the actual probability, if the system variables were to satisfy the strong law of large numbers (SLLN), which we do not assume here. From a quality of service perspective, it is desirable to keep $p_{n}^{o}$ to be less than some small upper bound $\eta_{n}^{o}$.

Our overall objective is to maximize the long-term average total sensing rate at all sensor nodes subject to the constraints on the "rate" of battery discharge for all nodes. With the above setting, we formulate the problem as follows:

$$
\begin{gathered}
\max _{\vec{P}, \vec{R}} \liminf _{T \rightarrow \infty} \frac{1}{T} \sum_{t=0}^{T-1} \sum_{n \in \mathcal{N}} R_{n}(t) \\
q_{n}^{d}(t+1)=\left(q_{n}^{d}(t)-\mu_{n}(\vec{P}(t))\right)^{+}+R_{n}(t), \\
q_{n}^{b}(t+1)=\min \left[q_{n}^{b}(t)-P_{n}(t)+r_{n}(t), B_{n}^{b}\right], \\
0 \leq P_{n}(t) \leq \min \left[q_{n}^{b}(t), P_{\text {peak }}\right], \\
R_{n}(t) \leq A_{n}(t), \\
\limsup _{T \rightarrow \infty} \frac{1}{T} \sum_{t=0}^{T-1} \sum_{n \in \mathcal{N}} q_{n}^{d}(t)<\infty \\
p_{n}^{o} \leq \eta_{n}^{o}, \forall n \in \mathcal{N} .
\end{gathered}
$$

In Problem (A), constraints (4) and (5) describe how the data and battery queues evolve, respectively. Constraints (6) are the energy conservation equations ensuring that we cannot oversubscribe the energy that is unavailable in the battery, nor can we exceed the peak power level. Constraints (7) are the rate conservation equations that bound the actual amount of sensed data $R_{n}(t)$ by the available amount of data $A_{n}(t)$. Constraint (8) is the QoS constraint for the data queue: we need to keep all the data queues stable. Constraint (9) is the QoS constraint that guarantees a low battery discharge rate for all nodes. 
Definition 2: We define virtual battery queues $\forall n \in \mathcal{N}$ :

$$
\begin{aligned}
\tilde{q}_{n}^{b}(t+1)= & \left(\left(\tilde{q}_{n}^{b}(t)-\eta_{n}^{o}\right)^{+}+I_{n}(t)-r_{n}(t)+M_{n}(t)\right. \\
& \left.+P_{n}(t)\right)^{+}
\end{aligned}
$$

where $M_{n}(t)=\left(q_{n}^{b}(t)-P_{n}(t)+r_{n}(t)-B_{n}^{b}\right)^{+}$is the amount of missed replenishment and

$$
I_{n}(t)= \begin{cases}0 & \text { if } P_{n}(t)=0 \text { or } q_{n}^{b}(t)-P_{n}(t)>P_{\text {peak }} \\ 1 & \text { otherwise }\end{cases}
$$

Theorem 1: If all the virtual battery queues $\tilde{q}_{n}^{b}(t)$ are strongly stable, i.e., $\lim \sup _{T \rightarrow \infty} \frac{1}{T} \sum_{t=0}^{T-1} \sum_{n} \tilde{q}_{n}^{b}(t)<\infty$, we then have $p_{n}^{o} \leq \eta_{n}^{o}, \forall n \in \mathcal{N}$.

The proof can be found in Appendix A. We give the main algorithm in the following section.

\section{Joint Rate Control and Power Allocation ALGORITHM}

Our algorithm is composed of two components: rate control and power allocation.

\section{Rate Control $(\mathrm{RC})$ :}

If $q_{n}^{d}(t) \leq \frac{V}{2}$, node $n$ chooses to sense all the available data, i.e., $R_{n}(t)=A_{n}(t)$; otherwise, reject all the data, i.e., $R_{n}(t)=0$. Here, $V \in(0, \infty)$.

\section{Power Allocation (PA):}

Solve

$$
\begin{aligned}
& \max _{\vec{P}(t)} \sum_{n}\left[q_{n}^{d}(t) \mu_{n}(\vec{P}(t))-\tilde{q}_{n}^{b}(t) P_{n}(t)\right] \\
& \text { s.t. } \quad 0 \leq P_{n}(t) \leq \min \left[q_{n}^{b}(t), P_{\text {peak }}\right],
\end{aligned}
$$

Equation (12) is a non-convex problem and the base station compute the solution in a centralized manner and allocate $\vec{P}(t)$.

It is clear that by using the $R C$, we make sure that the data queues remain within a certain bound and this has a positive effect on the batteries as well, since a portion of data is not allowed into the nodes. The natural question one would ask here is, whether $R C$ rejects too many packets in the first place to synthetically meet the constraints. In the following theorem, we show that this is not the case. We use the notation $y=$ $O(x)$ to represent $y$ going to 0 as $x$ goes to 0 .

Theorem 2: If the optimal instantaneous sensing rate vector is $\left\{\vec{R}^{*}(t), t \geq 0\right\}$ for any given sample $\{\vec{A}(t), t \geq 0\}$ and $\{\vec{r}(t), t \geq 0\}$, then the joint rate control $R C$ and power allocation $P A$ algorithm achieves:

$$
\begin{aligned}
q_{n}^{d}(t) & \leq \frac{V}{2}+A_{\max }, \\
\tilde{q}_{n}^{b}(t) & \leq \frac{g_{n}}{N_{n}}\left(\frac{V}{2}+A_{\max }\right),
\end{aligned}
$$

$$
\begin{aligned}
\sum_{n} & \left\{\liminf _{T \rightarrow \infty} \frac{1}{T} \sum_{t=0}^{T-1} R_{n}(t)\right\} \\
\geq \sum_{n} & \left\{\liminf _{T \rightarrow \infty} \frac{1}{T} \sum_{t=0}^{T-1} R_{n}^{*}(t)-\eta_{n}^{o}\left(\frac{g_{n}}{N_{n}}+\mu_{\text {max }}\left\lceil\frac{P_{\text {peak }}}{r_{\text {min }}}\right\rceil\right)\right. \\
& \left.-O\left(\frac{\left(\frac{1}{2} V-B_{n}^{b}\right)^{+}}{V}\right)\right\}
\end{aligned}
$$

where $\mu_{\max }=\max _{n} \log \left(1+\frac{g_{n} P_{\text {peak }}}{N_{n}}\right)$.

The detailed proof is in Appendix B. Observe Equation (15), the term $\eta_{n}^{o}\left(\frac{g_{n}}{N_{n}}+\mu_{\max }\left\lceil\frac{P_{\text {peak }}}{r_{\min }}\right\rceil\right)$ captures the influence of battery outage, and it is small since the battery outage threshold $\eta_{o}$ is usually set to be very small. The term $O\left(\frac{\left(\frac{1}{2} V-B_{n}^{b}\right)^{+}}{V}\right)$ represents the asymptotical property of the gap. One can observe that, by appropriately choosing the $V$-parameter, we can make the performance gap decay inversely proportional to the buffer sizes.

\section{NUMERICAL EXAMPLES}

We consider a base station and two sensor nodes in the numerical example. The simulation time is $T=10^{5}$ time slots. We use the rate power function given in Equation (1), where the background noise $N_{1}=0.01, N_{2}=0.02$, and the channel gains $g_{1}=0.5, g_{2}=1$. Each node is equipped with an infinite data buffer. The number of arrivals $A_{1}(t), t \geq 0$ and $A_{2}(t), t \geq 0$, are modeled as independent Poisson random variables with mean $\lambda_{1}=5, \lambda_{2}=10$ and $A_{\max }=30$. We set $\eta_{o}$, the threshold of battery outage probability to 0.03 and the peak power $P_{\text {peak }}=1.5 \mathrm{~W}$ for both nodes. The replenishment process we use for node 1 is i.i.d Bernoulli random variables Bernoulli (0.5) (i.e., $r_{1}(t)=1$ w.p. 0.5 and $r_{1}(t)=0$ w.p. 0.5 ) plus independent Gaussian noise with mean 0.2 and variance 0.01 (the average replenishment rate is then $\bar{r}_{1}=0.7$ ). We use for node 2 a replenishment process which is formed by a periodic deterministic sine waveform $\left(r_{\max }=2.5\right.$ and period 8000) plus independent Gaussian noise with mean 0.2 and variance 0.01, as shown in Figure 2 (a) (The cycles imitate the daily solar cycles for a solar battery and the average replenishment rate can be simply calculated $\bar{r}_{2}=1.03$ ). All the battery buffer sizes are set to be $B_{b}=50 \mathrm{~J}$.

We choose different values of the control parameter $V$ for the proposed algorithm and compare the results with the upper bound of optimal value. From Figure 2 (b), we see that as $V$ increases, the average total sensing rate of the algorithm keeps increasing and get closer to the upper bound. This is consistent with Equation (15). From Figure 2 (c), we see that as $V$ increases, the average data queue length (we here only plot the data queue length of node 1) keeps increasing but is upper bounded by the bound we get in Equation (13). This means the queueing delay increases as we improve the sensing rate, which can be viewed as a tradeoff. From Figure 2 (d) we observe that the upper bound of battery discharge probability (we only plot for node 1 here) also increases as $V$ increases but still bounded by the threshold. 


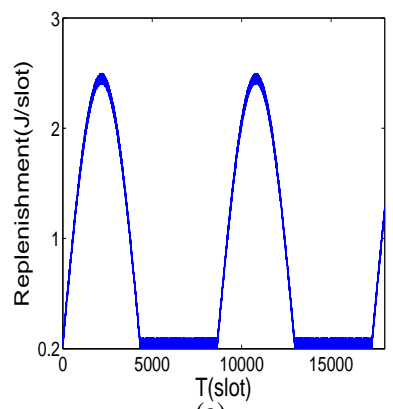

(a)

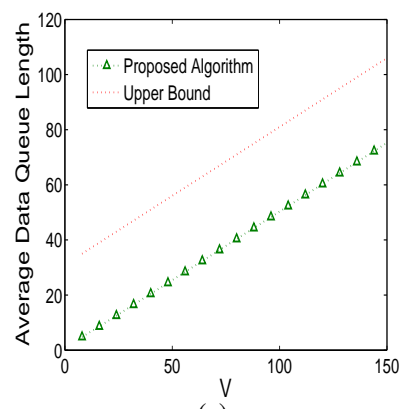

(c)

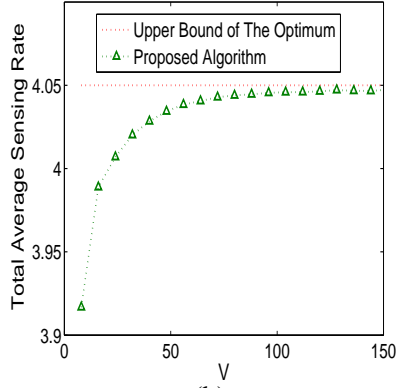

(b)

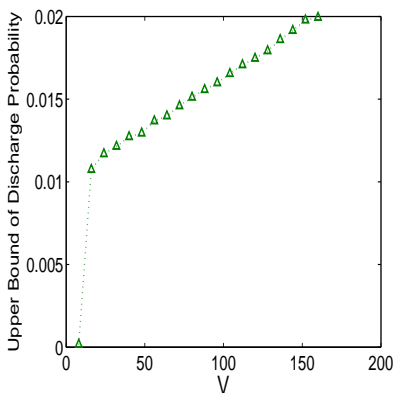

(d)
Fig. 2. (a) A sample replenishing process. Impact of the control parameter $V$ on (b) the average total sensing rate, (c) average data queue length, and (d) the battery discharge probability.

\section{CONCLUSION}

In this paper, we studied the problem of energy management in rechargeable wireless sensor networks under SINR interference model. Our objective was to maximize the average data sensing rate subject to QoS constraints on both data and battery queues. We developed a mathematical framework that handles the coupling between the data and battery queues. Unlike existing works, we allow for a the practical realities of having non-stationary replenishment and arrival processes. We develop a provably efficient algorithm that achieves the optimum solution within a small gap under the SINR interference model.

\section{REFERENCES}

[1] A. Mainwaring, J. Polastre, R. Szewczyk, D. Culler, and J. Anderson, "Wireless Sensor Networks for Habitat Monitoring," in Proc. of ACM WSNA, September 2002, pp. 88-97.

[2] N. Xu, S. Rangwala, K. Chintalapudi, D. Ganesan, A. Broad, R. Govindan, , and D. Estrin, "A Wireless Sensor Network for Structural Monitoring," in Proc. of SenSys, 2004, pp. 13-24.

[3] K. Martinez, R. Ong, and J. Hart, "Glacsweb: a Sensor Network for Hostile Environments," in Proc. of the 1st IEEE Communications Society Conference on Sensor and Ad Hoc Communications and Networks, Santa Clara, CA, 2004, pp. 81-87.

[4] J. Paradiso and M. Feldmeier, "A Compact, Wireless, Self-Powered Pushbutton Controller," in Proc. of the 3rd International Conference on Ubiquitous Computing, 2001, pp. 299-304.

[5] W. Weber, "Ambient Intelligence: Industrial Research on A Visionary Concept," in Proc. of the 2003 International Symposium on Low Power Electronics and Design, 2003, pp. 247-251.

[6] K. Kar, A. Krishnamurthy, and N. Jaggi, "Dynamic Node Activation in Networks of Rechargeable Sensors," IEEE/ACM Transactions on Networking, vol. 14, pp. 15-26, 2006.
[7] A. Kansal, J. Hsu, S. Zahedi, and M. Srivastava, "Power Management in Energy Harvesting Sensor Networks," ACM Transactions on Embedded Computing Systems, vol. 6, no. 4, September 2007.

[8] D. Niyato, E. Hossain, and A. Fallahi, "Sleep and Wakeup Strategies in Solar-Powered Wireless Sensor/Mesh Networks: Performance Analysis and Optimization," IEEE Transactions on Mobile Computing, vol. 6 , no. 2, pp. 221-236, February 2007.

[9] C. Vigorito, D. Ganesan, and A. Barto, "Adaptive Control of Duty Cycling in Energy-Harvesting Wireless Sensor Networks," in Proc. of IEEE SECON, 2007.

[10] M. Gatzianas, L. Georgiadis, and L. Tassiulas, "Control of Wireless Networks with Rechargeable Batteries," IEEE Transactions on Wireless Communications, vol. 9, no. 2, pp. 581-593, February 2010.

[11] Z. Mao, C. E. Koksal, and N. B. Shroff, "Near Optimal Power and Rate Control of Multi-hop Sensor Networks with Energy Replenishment: Basic Limitations with Finite Energy and Data Storage," to appear in IEEE/ACM Transactions on Automatic Control, 2012.

[12] M. Neely, "Energy Optimal Control for Time Varying Wireless Networks," IEEE Transactions on Information Theory, vol. 52, no. 7, pp. 2915-2934, July 2006.

[13] G. Foschini and Z.Miljanic, "A Simple Distributed Autonomous Power Control Algorithm and Its Convergence," IEEE Transactions on Vehicular Technology, vol. 42, no. 4, pp. 641-646, November 1993.

[14] T. Holliday, N. Bambos, A. Goldsmith, and P. Glynn, "Distributed Power Control for Time Varying Wireless Networks: Optimality and Convergence," in Proc. of Allerton Conference on Communication, Control and Computing, October 2003.

[15] Y. Shi, T. Hou, J. Liu, and S. Kompella, "How to Correctly Use the Protocol Interference Model for Multihop Wireless Networks," in Proc. of International Symposium on Mobile Ad Hoc Networking and Computing (MobiHoc), May 2009, pp. 239-248.

[16] M. Chiang and J. Bell, "Balancing Supply and Demand of Bandwidth in Wireless Cellular Networks: Utility Maximization over Power and Rates," in Proc. of IEEE Infocom, March 2004.

[17] D. O'Neil, D. Julian, and S. Boyd, "Adaptive Management of Network Resources," in Proc. of IEEE VTC, October 2003.

[18] P. Hande and M. Chiang, "Distributed Uplink Power Control for Optimal Sir Assignment in Cellular Data Networks," in Proc. of IEEE Infocom, April 2006.

\section{APPENDIX}

\section{A. Proof of Theorem 1}

Using the idea similar to [12], we have the fact that if any nonnegative queue represented with $Q(t)$ is strongly stable, then $\lim \sup _{T \rightarrow \infty} \frac{Q(T)}{T}=0$. Hence, if $\tilde{q}_{n}^{b}(t)$ is strongly stable, then $\lim \sup _{T \rightarrow \infty} \frac{\tilde{q}_{n}^{b}(T)}{T}=0$. From Equation (10), we have $\tilde{q}_{n}^{b}(t+1) \geq \tilde{q}_{n}^{b}(t)-\eta_{n}^{o}+P_{n}(t)-r_{n}(t)+M_{n}(t)+I_{n}(t)$. Note that $q_{n}^{b}(t+1)=q_{n}^{b}(t)-P_{n}(t)+r_{n}(t)-M_{n}(t)$. By summing from 0 to $T-1$, dividing by $T$ and taking limsup of both sides, we have $\lim \sup _{T \rightarrow \infty} \frac{\tilde{q}_{n}^{b}(T)}{T} \geq \lim _{T \rightarrow \infty} \frac{\tilde{q}_{n}^{b}(0)}{T}-$ $\eta_{n}^{o}+\lim \sup _{T \rightarrow \infty} \frac{1}{T} \sum_{t=0}^{T-1} I_{n}(t)+\lim _{T \rightarrow \infty} \frac{q_{n}^{b}(0)-q_{n}^{b}(T)}{T}$. Note that $\limsup _{T \rightarrow \infty} \frac{\tilde{q}_{n}^{b}(T)}{T}=0, \lim _{T \rightarrow \infty} \frac{\tilde{q}_{n}^{b}(0)}{T}=0$ $\lim _{T \rightarrow \infty} \frac{q_{n}^{b}(T)}{T}=0$, and $\lim _{T \rightarrow \infty} \frac{\tilde{q}_{n}^{b}(0)}{T}=0$. Combined with Equation (3) and (11), and the assumption that $r_{n}(t)>0, \forall t$, we get $p_{n}^{o}=\lim \sup _{T \rightarrow \infty} \frac{1}{T} \sum_{t=0}^{T-1} l_{n}^{o}(t) \leq$ $\lim \sup _{T \rightarrow \infty} \frac{1}{T} \sum_{t=0}^{T-1} I_{n}(t) \leq \eta_{n}^{o}, \forall n \in \mathcal{N}$.

\section{B. Proof of Theorem 2}

Proof of Equation (13): Since $A_{n}(t) \leq A_{\max }, \forall n \in \mathcal{N}, \boldsymbol{R C}$ is chosen to satisfy Equation (13).

Proof of Equation (14): Let $\Pi(t)=\left\{\vec{P}(t): 0 \leq P_{n}(t) \leq\right.$ $\left.\min \left[q_{n}^{b}(t), P_{\text {peak }}\right], \forall n\right\}$. Consider any node $n^{*} \in \mathcal{\mathcal { N }}$ and let $\overrightarrow{l^{*}}$ be the vector with 1 at $n^{*}$-th index and 0 for others. Since 
$\mu_{n^{*}}(\vec{P}(t))=\log \left(1+\frac{g_{n^{*}} P_{n^{*}}(t)}{N_{n^{*}}+\sum_{i \in N_{1}, i \neq n^{*}} g_{i} P_{i}(t)}\right) \leq \log (1+$ $\left.\frac{g_{n^{*}}}{N_{n^{*}}} P_{n^{*}}(t)\right) \leq \frac{g_{n^{*}}}{N_{n^{*}}} P_{n^{*}}(t)$, we then have

$$
\begin{aligned}
& \sum_{n}\left[q_{n}^{d}(t) \mu_{n}(\vec{P}(t))-\tilde{q}_{n}^{b}(t) P_{n}(t)\right] \\
\leq & \sum_{n}\left[q_{n}^{d}(t) \mu_{n}(\vec{P}(t)) 1_{\left[n \neq n^{*}\right]}-\tilde{q}_{n}^{b}(t) P_{n}(t)\right] \\
& +\frac{g_{n^{*}} q_{n^{*}}^{d}(t) P_{n^{*}}(t)}{N_{n^{*}}} .
\end{aligned}
$$

We now prove Equation (14) by induction. Without loss of generality, let $\tilde{q}_{n}^{b}(0) \leq \frac{g_{n}}{N_{n}}\left(\frac{V}{2}+A_{\max }\right), \forall n \in \mathcal{N}$. Suppose for all $t \geq 1, \tilde{q}_{n}^{b}(t-1) \leq \frac{g_{n}}{N_{n}}\left(\frac{V}{2}+A_{\max }\right), \forall n \in \mathcal{N}$ holds. In slot $t$, if node $n$ is assigned zero power, then by Equation (10) and (11) and the fact that $M_{n}(t) \leq r_{n}(t)$, we get $\tilde{q}_{n}^{b}(t) \leq \tilde{q}_{n}^{b}(t-1) \leq \frac{g_{n}}{N_{n}}\left(\frac{V}{2}+A_{\max }\right)$. Otherwise, consider any node $n^{*}$ and say $P_{n^{*}}(t)>0$. Since $\vec{P}(t)-P_{n^{*}}(t) \overrightarrow{l^{*}} \in \Pi(t)$, we then have from Equation (12) that $\sum_{n}\left[q_{n}^{d}(t) \mu_{n}(\vec{P}(t))-\right.$ $\left.\tilde{q}_{n}^{b}(t) P_{n}(t)\right] \geq \sum_{n \neq n^{*}}\left[q_{n}^{d}(t) \mu_{n}\left(\vec{P}(t)-P_{n^{*}}(t) \overrightarrow{l^{*}}\right)-\right.$ $\left.\tilde{q}_{n}^{b}(t) P_{n}(t)\right] \geq \sum_{n \neq n^{*}}\left[q_{n}^{d}(t) \mu_{n}(\vec{P}(t))-\tilde{q}_{n}^{b}(t) P_{n}(t)\right]$.

Combined with Equation (16), we have $\tilde{q}_{n^{*}}^{b}(t) \leq \frac{g_{n^{*}} q_{n^{*}}^{d}(t)}{N_{n^{*}}}$, and then $\tilde{q}_{n}^{b}(t) \leq \frac{g_{n} q_{n}^{d}(t)}{N_{n}} \leq \frac{g_{n}}{N_{n}}\left(\frac{V}{2}+A_{\max }\right)$.

Proof of Equation (15): Define $L\left(\vec{q}^{d}(t), \overrightarrow{\tilde{q}}^{b}(t)\right)=$ $\sum_{n}\left(q_{n}^{d}(t)\right)^{2}+\sum_{n}\left(\tilde{q}_{n}^{b}(t)\right)^{2}$, where $\vec{q}^{d}(t)$ and $\overrightarrow{\tilde{q}}^{b}(t)$ are the resulting queue dynamics of our algorithm given a sample path. Note that our problem is a sample path optimization problem, so $L\left(\vec{q}^{d}(t), \overrightarrow{\tilde{q}}^{b}(t)\right)$ is defined without conditional expectation. From Equation (4) and Equation (10), we have

$$
\begin{aligned}
& \Delta(t)=L\left(\vec{q}^{d}(t+1), \tilde{\tilde{q}}^{b}(t+1)\right)-L\left(\vec{q}^{d}(t), \tilde{\tilde{q}}^{b}(t)\right) \\
& \leq \sum_{n}\left[A_{\max }^{2}+\mu_{\max }^{2}\right]+\sum_{n}\left[\left(1+P_{\text {peak }}\right)^{2}+\left(\eta_{n}^{o}+r_{\max }\right)^{2}\right] \\
& +V \sum_{n} R_{n}(t)+2 \sum_{n}\left[q_{n}^{d}(t)-\frac{V}{2}\right] R_{n}(t) \\
& \quad-2 \sum_{n}\left[q_{n}^{d}(t) \mu_{n}(\vec{P}(t))-\tilde{q}_{n}^{b}(t) P_{n}(t)\right] \\
& +2 \sum_{n} \tilde{q}_{n}^{b}(t)\left[I_{n}(t)-r_{n}(t)+M_{n}(t)\right] .
\end{aligned}
$$

It is apparent that $R C$ is trying to minimize the term $\sum_{n}\left[q_{n}^{d}(t)-\frac{V}{2}\right] R_{n}(t)$, and $P A$ is trying to maximize the value of the term $\sum_{n}\left[q_{n}^{d}(t) \mu_{n}(\vec{P}(t))-\tilde{q}_{n}^{b}(t) P_{n}(t)\right]$. Since the optimal solution for Problem (A) may not be unique given a sample path, we let $\mathcal{P}^{*}$ be the optimal power solution set and $\vec{P}^{*} \in \mathcal{P}^{*}$ be any optimal solution, for Problem (A) given any sample path. Since the constraint set $\Pi(t)$ is queue dynamic related, it is possible that $\vec{P}^{*}(t) \notin \Pi(t)$.

Lemma 1: If by solving Equation (12), we get $\sum_{n}\left[q_{n}^{d}(t) \mu_{n}(\vec{P}(t))-\tilde{q}_{n}^{b}(t) P_{n}(t)\right]<\sum_{n}\left[q_{n}^{d}(t) \mu_{n}\left(\vec{P}^{*}(t)\right)-\right.$ $\left.\tilde{q}_{n}^{b}(t) P_{n}^{*}(t)\right]$, then there exists at least one node $\tilde{n}$ such that $I_{\tilde{n}}\left(t^{\prime}\right)=1$ for some $t^{\prime} \leq t$ and $t-t^{\prime} \leq\left\lceil\frac{P_{\text {peak }}}{r_{\text {min }}}\right\rceil$.
Proof: Without loss of generality, we assume the initial battery state satisfy $q_{n}^{b}(0) \geq P_{\text {peak }}, \forall n \in \mathcal{N}$.

If $q_{n}^{b}(t)<P_{\text {peak }}$ for some user node $\tilde{n}$, then by Equation (11), it means $I_{\tilde{n}}\left(t^{\prime}\right)=1$ for $\tilde{n}$ and some $t^{\prime} \leq t$ where $t^{\prime}$ is the largest time no larger than $t$ such that $I_{\tilde{n}}\left(t^{\prime}\right)=1$. We show $t-t^{\prime} \leq\left\lceil\frac{P_{\text {peak }}}{r_{\text {min }}}\right\rceil$ by contradiction. Suppose $t-t^{\prime}>\left\lceil\frac{P_{\text {peak }}}{r_{\text {min }}}\right\rceil$, then it means $I_{\tilde{n}}(\tau)=0, \forall \tau \in\left[t^{\prime}, t\right]$. This means ${ }^{r_{\min }} P_{\tilde{n}}(\tau)=0, \forall \tau \in\left[t^{\prime}, t\right]$, otherwise it will contradict $t^{\prime}$ being the largest time no larger than $t$ such that $I_{\tilde{n}}\left(t^{\prime}\right)=1$. Since $r_{\tilde{n}}(t) \geq r_{\min }$, if $P_{\tilde{n}}(\tau)=0, \forall \tau \in\left[t^{\prime}, t\right]$, then $q_{\tilde{n}}^{b}(t) \geq q_{\tilde{n}}^{b}\left(t^{\prime}\right)+\left(t-t^{\prime}\right) r_{\text {min }}>q_{\tilde{n}}^{b}\left(t^{\prime}\right)+P_{\text {peak }} \geq P_{\text {peak }}$ which contradicts $q_{\tilde{n}}^{b}(t)<P_{\text {peak }}$.

If $q_{n}^{b}(t) \geq P_{\text {peak }}, \forall n \in \mathcal{N}$. $P A$ is then equivalent to solving

$$
\begin{array}{ll}
\max _{\vec{P}(t)} \sum_{n}\left[q_{n}^{d}(t) \mu_{n}(\vec{P}(t))-\tilde{q}_{n}^{b}(t) P_{n}(t)\right] \\
\text { s.t. } & 0 \leq P_{n}(t) \leq P_{\text {peak }}
\end{array}
$$

which means $\sum_{n}\left[q_{n}^{d}(t) \mu_{n}(\vec{P}(t))-\tilde{q}_{n}^{b}(t) P_{n}(t)\right] \geq$ $\sum_{n}\left[q_{n}^{d}(t) \mu_{n}\left(\vec{P}^{*}(t)\right)-\tilde{q}_{n}^{b}(t) P_{n}^{*}(t)\right]$.

By Lemma 1, Equation (13) and (14), we then have

$$
\begin{aligned}
& \Delta(t)=L\left(\vec{q}^{d}(t+1), \overrightarrow{\tilde{q}}^{b}(t+1)\right)-L\left(\vec{q}^{d}(t), \overrightarrow{\tilde{q}}^{b}(t)\right) \\
& \leq \sum_{n}\left[A_{\max }^{2}+\mu_{\max }^{2}\right]+\sum_{n}\left[\left(1+P_{\text {peak }}\right)^{2}+\left(\eta_{n}^{o}+r_{\max }\right)^{2}\right] \\
& +V \sum_{n} R_{n}(t)+2 \sum_{n}\left[q_{n}^{d}(t)-\frac{V}{2}\right] R_{n}^{*}(t) \\
& \quad-2 \sum_{n}\left[q_{n}^{d}(t) \mu_{n}\left(\vec{P}^{*}(t)\right)-\tilde{q}_{n}^{b}(t) P_{n}^{*}(t)\right] \\
& +2 \sum_{n} \tilde{q}_{n}^{b}(t)\left[I_{n}(t)-r_{n}(t)+M_{n}(t)\right] \\
& +2 \mu_{\text {max }}\left\lceil\frac{P_{\text {peak }}}{r_{\text {min }}}\right\rceil\left(\frac{V}{2}+A_{\max }\right) \sum_{n} I_{n}(t) .
\end{aligned}
$$

Using similar arguments as in [11], we have $\frac{1}{V} \lim \sup _{T \rightarrow \infty} \frac{1}{T} \sum_{t=0}^{T-1} \sum_{n} \tilde{q}_{n}^{b}(t)\left[P_{n}^{*}(t)-r_{n}(t)\right] \leq$ $O\left(\frac{1}{V}\right), \quad \frac{1}{V} \liminf _{T \rightarrow \infty} \frac{1}{T} \sum_{t=0}^{T-1} \sum_{n} \tilde{q}_{n}^{b}(t) M_{n}(t) \leq$ $\sum_{n} O\left(\frac{\left(\frac{1}{2} V-B_{n}^{b}\right)^{+}}{V}\right)$, and $\frac{1}{V} \lim \sup _{T \rightarrow \infty} \frac{1}{T} \sum_{t=0}^{T-1} \sum_{n} q_{n}^{d}(t)\left[R_{n}^{*}(t)-\right.$ $\left.\mu_{n}\left(\vec{P}^{*}(t)\right)\right] \leq O\left(\frac{1}{V}\right)$.

By summing from 0 to $T-1$, dividing by $T$ and $V$, taking $\liminf _{T \rightarrow \infty}$ for Equation (17), we get

$$
\begin{aligned}
& \sum_{n}\left\{\liminf _{T \rightarrow \infty} \frac{1}{T} \sum_{t=0}^{T-1} R_{n}(t)\right\} \\
& \geq \sum_{n}\left\{\liminf _{T \rightarrow \infty} \frac{1}{T} \sum_{t=0}^{T-1} R_{n}^{*}(t)-\eta_{n}^{o}\left(\frac{g_{n}}{N_{n}}+\mu_{\text {max }}\left\lceil\frac{P_{\text {peak }}}{r_{\text {min }}}\right\rceil\right)\right. \\
&\left.\quad-O\left(\frac{\left(\frac{V}{2}-B_{n}^{b}\right)^{+}}{V}\right)\right\} .
\end{aligned}
$$

